\title{
Thought control with the dopamine transient
}

\author{
Mihaela D. Iordanova ${ }^{1}$ (C) \\ Published online: 18 October 2018 \\ (C) Psychonomic Society, Inc. 2018
}

\section{Summary}

Fine-tuning and coordinating neural activity is essential for an efficient brain. Athalye and colleagues (2018) provide important evidence that neural patterns can be streamlined by inducing dopamine transients.

Keywords Reinforcement learning $\cdot$ Brain stimulation $\cdot$ Reward $\cdot$ Motor cortex

It has been almost a century since Thorndike placed cats in puzzle boxes and watched them learn to escape through trial and error. Once learned, environmental stimuli would elicit the appropriate response leading to the desirable outcome of freedom. The role of the outcome, Thorndike famously proposed, was to "stamp in" the stimulus-response association and thus increase the probability of the response being repeated, but without representing the outcome in the associative structure. To put it another way, the animal reflexively worked towards a goal without explicitly thinking about it. Although not sufficient, this law of effect has been used to account for many actions that comprise the behavioral repertoire of animals and humans. More recently in my own household, I used Thorndikean principles to tackle the monumental task of potty training a toddler. A pee in the potty earned my daughter a single M\&M. It worked like a charm.

In a classic operant experiment an action such as a lever press is repeated if it is reinforced with a desirable outcome. Interestingly, we have known for some time that actions, and by extension their underlying neural representations, can be reinforced not only with physical rewards but also with brain stimulation (Olds \& Milner, 1954). That is, reinforcement can control our behavior and indirectly our thoughts. But it is not until now that we have evidence of neural reinforcement shaping neuronal patterns, bypassing environmental input.

Athalye, Santos, Carmena, and Costa (2018) designed an ingenious experiment that sought to determine whether neural reinforcement can control the production of specific patterns

Mihaela D. Iordanova

mihaela.iordanova@concordia.ca

1 Department of Psychology, Center for Studies in Behavioral Neurobiology, Concordia University, Montreal, Québec, Canada of neuronal activity. In their experiment, the authors recorded neuronal activity in the primary motor cortex (M1) and isolated neural patterns or states defined by the relative firing rates of individual neurons. Neuronal activity within two distinct ensembles were translated into auditory feedback (tones) of different frequencies, so the occurrence of these tones became a read-out of the neural activity. Using closed-loop stimulation, a specific pattern of neuronal activity within an ensemble associated with a tone of a chosen frequency (target tone) triggered the delivery of a reinforcing optical stimulation. This optical stimulation used channelrhodopsin to activate tyrosine hydroxylase positive $(\mathrm{Th}+)$ neurons in the Ventral tegmental area (VTA) in Th-cre mice. In their paper, this optical stimulus had reinforcing effects on behavior. The neural pattern that generated the frequency of the target tone paired with neural reinforcement became more frequent, resulting in the target tone occurring more often across the session compared to the non-reinforced target. That is, reinforced neural patterns in the motor cortex were re-entered into more often, just like reinforced behavioral responses.

Of course, some actions are more similar than others. A good tennis player is more likely to be good at badminton than basketball. Similar actions have more similar neural activity (Georgopoulos, Kalaska, Caminiti, \& Massey, 1982). Athalye et al. (2018) reported that the neural activity in the motor cortex produced an increase in the occurrence of tones of similar frequencies to that of the reinforced target. That is, neural reinforcement stamps in the re-entry not only of the paired neural activity pattern but also generalizes to "similar" neural patterns within M1.

Generalization occurs not only at the level of the action but also at the level of the stimuli that invigorate those actions. Potty training readily transfers to the toilet. Untrained stimuli similar to the trained stimulus can initiate lever press. 
Importantly, the process of generalization is present beyond stimulus or action similarity. Associative links provide another way through which reinforcement of one stimulus transfers to another. In sensory pre-conditioning, two neutral stimuli are presented together, e.g., a tone and a light. Subsequently, one of the stimuli (i.e., the light) is paired with a desirable outcome. Interestingly, this learning is transferred to the stimulus that was never explicitly paired with the outcome (i.e., the tone). Indeed, VTA dopamine transients support exactly this form of learning, namely sensory pre-conditioning of two neutral cues (Sharpe et al., 2017). The next question is whether the co-occurrence of neural activity patterns across distinct ensembles can lead to increased re-entry of one of those patterns if the other received reinforcement, and whether this can happen across brain areas.

And it need not stop here. Generalization can also be a product of categorization where stimuli with similar predictive histories are grouped together. Newly acquired information about one exemplar readily transfers or generalizes to another exemplar within the same category (Honey \& Hall, 1989). That is, different neural patterns reinforced by VTA DA transients at different time points may become grouped such that subsequent modulation through experience of one neural pattern may translate into similar modulation of another pattern within the same category in the absence of direct experience. This offers a tractable way for the field to think about the dynamics between neuronal ensemble activity and the information it represents across the spectrum of similarity and time.

How is neural reinforcement able to shape the firing pattern of distinct neurons in M1? Athalye et al. (2018) argued that the extent to which these neurons share a common input is critical in the process. A shared input increases the covariance among the neurons comprising the ensemble across learning episodes. That is, as learning progresses, the firing rate of one neuron in the ensemble will more tightly relate to the firing rate of another neuron within the same ensemble. To test this, the authors partitioned the variance of neural firing using factor analysis into individual and shared input, with the former driving neural activity of individual neurons in an uncoordinated fashion and the latter coordinating the ensemble activity. They reported that the shared covariance increased with learning and aligned with that specified by their decoder. This was evidenced at the individual level in good learners, but not in poor learners, with the latter showing greater frequency of the target tone over the non-target at the population but not at the individual level. Further, this coordinated neural activity was present in the ensemble responsible for generating the target tone but not in other irrelevant-to-the-task ensembles, and not at the level of the firing properties of individual neurons.

So, why should reinforcement drive increase in correlated activity across neurons? One possibility is that reinforcement is involved in the recruitment of neurons and the synchronisation of neuronal activity within an ensemble. The greater the number of coordinated neural activity within an ensemble the greater the control over behavior or the more vigorous the action.

Fine-tuning neuronal patterns through reinforcement by increasing coordinated neural activity may also serve to increase the signal-to-noise ratio. The process of fine-tuning is clear in behavior. Initially, reinforcement is used to shape animal behavior by reinforcing actions that increasingly resemble the target action. Once the target action is achieved, reinforcement becomes action-specific and irrelevant behaviors are minimized. A well-trained animal is efficient in obtaining a goal. My daughter earned M\&Ms across a variety of behaviors that slowly moved her towards her goal, to finally achieve the most optimized action. Reinforcement increases covariance among neurons within an ensemble in order to promote efficiency in the neural activity, maximize relevant and minimize irrelevant activity. However, this efficiency or streamlined re-entry into reinforced neural patterns may also be the basis of maladaptive thoughts or behaviors. Positive or negative reinforcement could trigger rumination or intrusive thought patterns driving anxiety and addiction.

The law of effect is one of the simplest, most rudimentary accounts of associative learning. Yet, its employment in the present context has pushed the boundaries of our understanding of its underlying neural dynamics. Using more complex models that incorporate the richness of the associative tapestry, from forming rich representations of individual events to complex hierarchical relationships, we are bound to further enhance our knowledge of how neural dynamics are shaped by reinforcement. Indeed, recent research into the role of mesolimbic DA in prediction error supports the idea that optical stimulation of Th+ neurons in the VTA does not merely stamp in responses (neural or behavioral) or value, but rather serves to construct a complex and detailed representation of the world.

\section{References}

Athalye VR, Santos FJ, Carmena JM, Costa RM. (2018) Evidence for a neural law of effect. doi: https://doi.org/10.1126/science.aao6058.

Georgopoulos AP, Kalaska JF, Caminiti R, Massey JT. (1982) On the relations between the direction of two-dimensional arm movements and cell discharge in primate motor cortex. Journal of Neuroscience, 2(11), 1527-37

Honey RC, \& Hall G. (1989) Acquired equivalence and distinctiveness of cues. Journal of Experimental Psychology: Animal Behavioral Processes, 15(4), 338-46

Olds, J., \& Milner, P., (1954) Positive reinforcement produced by electrical stimulation of septal area and other regions of rat brain, Journal of Comparative and Physiological Psychology, 47, 419-427

Sharpe MJ, Chang CY, Liu MA, Batchelor HM, Mueller LE, Jones JL, Niv Y, Schoenbaum G. (2017) Dopamine transients are sufficient and necessary for acquisition of model-based associations. Nature Neuroscience 20(5), 735-742. doi: https://doi.org/10.1038/nn.4538 